\title{
An algorithm for estimating migration spatial resolution in layered media with focal beams
}

\author{
Bangrang $\mathrm{Di}^{1}$, Sangxu Wang ${ }^{1}$, Xiang-Yang $\mathrm{Li}^{2}$ and David Booth ${ }^{2}$ \\ ${ }^{1}$ CNPC Geophysical Key Laboratory, China University of Petroleum, Beijing, 102249, China \\ ${ }^{2}$ British Geological Survey, West Mains Road, Edinburgh, Scotland, UK, EH9 3BG
}

Running title: Estimating migration spatial resolution in layered media

Submitted on 10 December 2005

Re-submitted on 17 March 2006

$1^{\text {st }}$ Revised on $\quad 17$ August 2006

2nd Revised on $\quad 23$ February 2007

Address for correspondence

Professor Bangrang Di

P.O. Box 902

20 Xueyuan Road

Beijing, 100083

China

E-mail: dibr@cup.edu.cn or dibangr@yahoo.com 


\begin{abstract}
Research on seismic resolution has recently progressed from studying separate vertical and horizontal resolution to general spatial resolution. However, most studies on the spatial resolution are limited to a homogeneous medium and thus are not applicable to layered or complex media. We have filled this gap by developing a numerical algorithm for estimating spatial resolution in layered media. Using Berkhout's focusing approach, we extrapolate the wavefield with Rayleigh Integration and calculate the spatial resolution of layered media using a recursive numerical method. This makes it possible to calculate the spatial resolution for different imaging planes, corresponding to different dip angles and acquisition geometries. This numerical scheme has been tested on a homogenous model to verify its accuracy, and applied to a three-layer physical model. Results show that the algorithm presented here provides an effective method for designing seismic acquisitions and for analyzing the spatial resolution of seismic imaging algorithms.
\end{abstract}

\title{
Keywords:
}

double focusing imaging, spatial resolution, migration, wave field extrapolation, focal beams, acquisition design

\section{Introduction}

One of the primary purposes of seismic migration is to enhance the spatial resolution of seismic images, and so improve the interpretation of seismic data. For the purposes of reservoir characterization and lithology prediction, seismic images with higher resolution can provide more reliable information for the positioning of faults, pinches and overlaps, which in turn helps to locate hydrocarbon traps more accurately. From the 1980s, research on improving seismic resolution on both vertical and horizontal has attracted considerable and continuous interests, and has progressed from the vertical resolution in the time domain to the resolution in spatial domain for seismic migration and imaging.

There are two main theories concerning the spatial resolution of seismic migration. The first is Beylkin's (1985) raytracing-based inversion method which utilizes the generalized Radon transform to calculate the spatial resolution (e.g. Von Seggern 1994, Vermeer 1999, Chen and Schuster 1999, and others). The second is Berkhout's (1997) Kirchhoff-integration 
based method which uses a double dynamic focusing algorithm with focal beams (e.g. Wapenaar 1997, Berkhout et al. 2001, Volker et al. 2001, and others). Ma et al. (2002) derived a formula to calculate the spatial resolution of each trace in prestack or poststack gathers according to the quarter-period (or quarter wavelength) criteria ( $T / 4$ or $\lambda / 4$ ).

However, all these methods are restricted to a homogeneous medium; this represents the ideal case and substantially differs from reality. In this paper we follow Berkhout's (1997) approach with focal beams and derive a method for estimating spatial resolution in layered media. Our method includes two steps. In the first step, for a given layered model and acquisition geometry, we derive the focused source beams and focused detector beams from a point diffractor by forward and inverse extrapolation of the wavefield through the layer interfaces. These beams will in turn construct a migrated/focused image of the point diffractor, which is employed as a digital resolution function. In the second step, we calculate the spatial resolution for various imaging planes with different dip angles in the resolution function, followed by evaluating the effects of dip and acquisition on migration resolution. With these two steps we extend the calculation of spatial resolution of seismic migration from a homogeneous medium to layered media.

\section{Theory and Method}

\subsection{Definition of the resolution function of double focusing migration}

Berkhout (1997) presented a comprehensive theory of imaging of acoustic energy by wave field extrapolation using a double focusing approach. Our work on spatial resolution will follow that approach. We assume that a subsurface reflector is buried at depth $Z_{m}$, and that an array of sources and detectors (receivers) are placed on the surface $Z_{0}$. According to Berkhout (1997), the primary reflection from the interface $Z_{m}$ as recorded at the surface $Z_{0}$ can be written as, in the frequency domain,

$$
P_{\left(Z_{0}\right)}=D_{\left(Z_{0}\right)} W_{D\left(Z_{0}, Z_{m}\right)} R_{\left(Z_{m}\right)} W_{S\left(Z_{m}, Z_{0}\right)} S_{\left(Z_{0}\right)}
$$

where $P_{\left(\mathrm{Z}_{0}\right)}$ denotes the data matrix (seismic records). $W_{D\left(Z_{0}, Z_{m}\right)}$ and $W_{S\left(Z_{0}, Z_{m}\right)}$ represent the upward and downward propagation matrices, respectively; $R_{\left(Z_{m}\right)}$ denotes the reflectivity matrix; $D_{\left(z_{0}\right)}$ and $S_{\left(Z_{0}\right)}$ denote the receiver and source sampling matrices, 
respectively, which can both be normalized into unit matrices.

We denote the detector (receiver) focusing operator as $F_{D\left(z_{m}, z_{0}\right)}$ and the source focusing operator as $F_{S\left(Z_{0}, Z_{\mathrm{m}}\right)}$. Following Berkhout (1997), the process of double focusing migration may be described as:

$$
\Delta P_{\left(Z_{m}\right)}=F_{D\left(Z_{m}, Z_{0}\right)} P_{\left(Z_{0}\right)} F_{S\left(Z_{0}, Z_{m}\right)}
$$

where $\Delta P_{\left(Z_{m}\right)}$ represents the subsurface image. The focal detector and source beams are respectively defined by the following formulae:

$$
\begin{aligned}
& B_{D\left(Z_{m}, Z_{0}\right)}=F_{D\left(Z_{m}, Z_{0}\right)} D_{\left(Z_{0}\right)} W_{D\left(Z_{0}, Z_{m}\right)}=F_{D\left(Z_{m}, Z_{0}\right)} W_{D\left(Z_{0}, Z_{m}\right)} \\
& B_{S\left(Z_{0}, Z_{m}\right)}=W_{S\left(Z_{m}, Z_{0}\right)} S_{\left(Z_{0}\right)} F_{S\left(Z_{0}, Z_{m}\right)}=W_{S\left(Z_{m}, Z_{0}\right)} F_{S\left(Z_{0}, Z_{m}\right)} .
\end{aligned}
$$

Substituting equations (1), (3) and (4) into equation (2) gives,

$$
\Delta P_{\left(Z_{m}\right)}=B_{D\left(Z_{m}, Z_{0}\right)} R_{\left(Z_{m}\right)} B_{S\left(Z_{0}, Z_{m}\right)}
$$

According to Berkhout (1985), the conditions for an ideal migration are,

$$
\begin{aligned}
& B_{D\left(Z_{M}, Z_{0}\right)}=B_{D O\left(Z_{M}, Z_{0}\right)}=F_{D O\left(Z_{M}, Z_{0}\right)} W_{D\left(Z_{0}, Z_{\mathrm{m}}\right)}=\mathbf{I} \text { and } \\
& B_{S\left(Z_{0}, Z_{m}\right)}=B_{S O\left(Z_{0}, Z_{m}\right)}=W_{S\left(Z_{m}, Z_{0}\right)} F_{S O\left(Z_{0}, Z_{m}\right)}=\mathbf{I}
\end{aligned}
$$

where $\mathbf{I}$ is the unit matrix; $B_{D O\left(Z_{M}, Z_{0}\right)}$ and $B_{S O\left(Z_{0}, Z_{m}\right)}$ are ideal focal beams; $F_{D O\left(z_{M}, z_{0}\right)}$ and $F_{S O\left(z_{0}, z_{m}\right)}$ are ideal focusing operators that are in fact the inverse of $W_{D\left(Z_{0}, Z_{\mathrm{m}}\right)}$ and $W_{S\left(Z_{m}, Z_{0}\right)}$ respectively.

Inserting equations (6) and (7) into (5) gives,

$$
\Delta P_{\left(\mathrm{Z}_{\mathrm{m}}\right)}=R_{\left(\mathrm{Z}_{\mathrm{m}}\right)}
$$

which represents the result of an ideal double focusing migration where the imaging result is the reflectivity matrix. Equation (8) indicates that the ideal migration occurs when the focus point (or imaging point) coincides with the reflection point (the target point). In this case, the migration resolution function is an impulse function. However, in reality, the estimated 
focusing operators are often approximations of the ideal case, and cannot fully compensate for the effect of the surface sampling and the subsurface propagation. Therefore, the imaging point may deviate from the target point, introducing error to the imaging result. The resolution function describes the deviation of the imaging point from the target point, and it can be formulated as:

$$
R F_{\mathrm{d}\left(\mathrm{z}_{\mathrm{m}}\right)}=\sum_{i} B_{\operatorname{Did}\left(Z_{m}, Z_{0}\right)} B_{\operatorname{Sdi}\left(Z_{0}, Z_{m}\right)}
$$

where $R F_{\mathrm{d}\left(\mathrm{z}_{\mathrm{m}}\right)}$ is the resolution function for a given target point $d$ at depth $\mathrm{z}_{\mathrm{m}}$, and $\Sigma$ denotes the summation over all possible imaging points (i) of different acquisition geometries within the focusing aperture; $B_{\text {Did }}$ is the focal detector beam between target point $d$ and imaging point $i ; B_{\mathrm{Sid}}$ is the corresponding focal source beam between the two points; the resolution function reaches its maximum when $i$ equals $d$. Equation (9) indicates that the focal beams are the key factors determining the resolution function.

\subsection{Calculating the resolution function in a homogeneous medium}

Volker et al. (2001) discussed how to calculate the focusing resolution function for a point diffractor in a homogeneous medium. As shown in Figure 1, considering the pressure wave field generated from a dipole source, the propagation operator can be written as the complex function:

$$
W=\frac{1}{2 \pi} \frac{1+j k r_{\mathrm{d}}}{r_{\mathrm{d}}^{2}} \cos \varphi_{d} e^{-j k r_{d}}
$$

where $r_{d}$ is the distance from the diffractor to the detector; $\varphi_{\mathrm{d}}$ is the emergent angle at the diffractor; wavenumber, $k=\omega / \mathrm{v}$ where $\omega$ and $\mathrm{v}$ are the circular frequency and the medium velocity, respectively. The diffractor in this case represents the target point $(d)$ in equation (9).

The focusing operator is defined as the complex conjugate of $W$ :

$$
F=\frac{1}{2 \pi} \frac{1-j k r}{r^{2}} \cos \varphi e^{j k r}
$$

where $\varphi$ denotes the emergence angle from the detector to the imaging point and $r$ denotes 
the distance from the imaging point to the detector. Here, the imaging point is the same as the imaging point (i) in equation (9). Thus, the focal detector beam and focal source beam can be written as:

$$
\begin{aligned}
& B_{D(x, y, z, \omega)}=\frac{k^{2}}{4 \pi^{2}} \iint_{L x L y} \frac{\cos \varphi_{i}}{r_{d}} e^{-j k r_{i}} \frac{\cos \varphi}{r} e^{j k r} S_{D\left(x_{D}, y_{D}, z_{D}\right)} d x_{D} d y_{D} ; \\
& B_{S(x, y, z, \omega)}=\frac{k^{2}}{4 \pi^{2}} \iint_{L x L y} \frac{\cos \varphi_{i}}{r_{d}} e^{-j k r_{i}} \frac{\cos \varphi}{r} e^{j k r} S_{S\left(x_{s}, y_{s}, z_{s}\right)} d x_{S} d y_{S} .
\end{aligned}
$$

In equation (12) and (13), $S_{D\left(x_{D}, y_{D}, z_{D}\right)}\left(S_{S\left(x_{S}, y_{S}, z_{S}\right)}\right)$ represents the detector (source) sampling operator, the value of which is 1 at the position of the detector (source) point and zero at other positions; 2Lx and 2Ly define the extrapolation aperture. Once the focal beams are determined, the resolution function can be calculated using equation (9).

Once the resolution function is determined, the corresponding spatial resolution of the migrated image is defined as the half width of the dominant peak in the resolution function.

\subsection{The theoretical basis for wave field extrapolation in layered media}

The integral solution to the wave equation is the theoretical basis for wave field extrapolation in layered stratified media (Berkhout 1985). The Kirchhoff integral solution is often used for curved interfaces, whilst Rayleigh integration is used for planar interfaces. Here, we consider only horizontal or dipping planar interfaces with a dipole source, and thus Rayleigh integration II (Berkhout 1985) is used.

As shown in Figure 2, let $\left(0,0,-z_{B}\right)$ denote the coordinates of point $B$ representing the source location, and $\left(\mathrm{o}, \mathrm{o}, \mathrm{z}_{\mathrm{A}}\right)$ denote the coordinates of point $\mathrm{A}$ at which the wavefield needs to be extrapolated. Point A and point $B$ are placed symmetrically with respect to the reference plane $S_{1}$. The dipole at point $B$ is chosen to be a pressure source with unit amplitude and single frequency.

Firstly, the pressure field at point A generated from the dipole source at point B can be directly calculated using the propagation operator $\mathrm{W}$ in equation (10). Denoting this calculated pressure wave at point $\mathrm{A}$ as $\mathrm{P}_{\mathrm{A} 0}$, it follows (Volker et al. 2001),

$$
P_{A 0}=\frac{1+j k\left(z_{A}+z_{B}\right)}{2 \pi\left(z_{A}+z_{B}\right)} e^{-j k\left(z_{A}+z_{B}\right)} \approx \frac{j k}{2 \pi\left(z_{A}+z_{B}\right)} e^{-j k\left(z_{A}+z_{B}\right)}
$$


assuming $k r>>1$.

Secondly, the pressure field at point A can also be obtained by extrapolating the wavefield through the reference plane $S_{1}$ using Rayleigh integration II. Denoting the extrapolated wavefield at point $\mathrm{A}$ as $\mathrm{P}_{\mathrm{A} 1}$ and following the derivation in the Appendix,

$$
P_{\mathrm{A} 1}=\frac{j k}{2 \pi\left(z_{A}+z_{B}\right)}\left[e^{-j k\left(z_{A}+z_{B}\right)}-\frac{Z_{A} Z_{B}\left(Z_{A}+Z_{B}\right)}{r_{A m} r_{B m}\left(r_{A m}+r_{B m}\right)} e^{-j k\left(r_{A m}+r_{B m}\right)}\right],
$$

where $r_{A m}=\sqrt{Z_{A}{ }^{2}+L^{2}}, r_{B m}=\sqrt{Z_{B}{ }^{2}+L^{2}}$. When $L \rightarrow \infty$, that is, the area of plane $\mathrm{S}_{1}$ is $\infty, P_{A 1}=P_{A 0}$. This indicates that the result of the indirect extrapolation is equal to that of direct calculation, which forms the basis of wave field extrapolation in layered stratified media.

\subsection{Calculating the resolution function in layered media}

We follow a recursive numerical scheme to calculate the resolution function for double focusing migration in layered media. Each individual layer can be considered as a homogeneous medium. Therefore, the propagation operator in equation (9) and the focusing operator in equation (10) for a homogeneous medium can then be used to extrapolate the wavefield within the layer. This can be applied recursively layer by layer, making it possible to extrapolate the wave field in the forward and inverse directions through all the layers from the surface to the diffractor using the integral solutions of the wave equation.

We denote the depth of the surface as $Z_{0}$, and the depths of the layer interfaces as $z_{1}, \quad z_{2} \cdots z_{m}$ respectively. The interfaces of the layers are divided into grids (Figure 1 ). The deepest layer interface is the focusing plane, or imaging plane. This imaging plane may have an arbitrary dip angle. The different dip angles correspond to the spatial resolution in different directions. The target diffractor locates at point $d$, the middle of the imaging plane, as in the case of Figure 1; the imaging point position (i) varies on the imaging plane, and under this setup, the imaging result represents the resolution function.

As shown in equation (9), calculation of the focal beams is the key for determining the resolution function. Next, we discuss the procedure to calculate the focal detector beams, and the focus source beams can be calculated in the same fashion. Figure 3 illustrates this process. 
Firstly, as shown Figure 3a, we extrapolate the pressure wave field recursively through the interfaces upward from the diffractor to the detector position $P_{D\left(x_{0}, y_{0}, z_{0}\right)}$ on the surface, as the forward focusing step. Starting at interface $Z_{m}$ leads to,

$$
\begin{aligned}
& Z_{m-1}: P_{D\left(x_{m-1}, y_{m-1}, z_{m-1}\right)}=P_{D d\left(Z_{m}\right)} W_{D\left(z_{m-1}, z_{m}\right)} \\
& Z_{m-2}: P_{D\left(x_{m-2}, y_{m-2}, z_{m-2}\right)}=\iint P_{D\left(x_{m-1}, y_{m-1}, z_{m-1}\right)} W_{D\left(z_{m-2}, z_{m-1}\right)} S_{\left(x_{m-1}, y_{m-1}, z_{m-1}\right)} d x d y \\
& Z_{0}: \text { at the surface } P_{D\left(x_{0}, y_{0}, z_{0}\right)}=\iint P_{D\left(x_{1}, y_{1}, z_{1}\right)} W_{D\left(z_{0}, z_{1}\right)} S_{D\left(x_{1}, y_{1}, z_{1}\right)} d x d y
\end{aligned}
$$

Secondly, as shown in Figure 3b, we extrapolate the wavefield $P_{D\left(x_{0}, y_{0}, z_{0}\right)}$ recursively downward through the layer interfaces to the imaging point $i$, which varies around the diffractor $d$ on the imaging plane. This is the inverse focusing step. Starting at the surface $z_{0}$, gives,

$$
\begin{gathered}
z_{1}: \quad B_{D\left(x_{1}, y_{1}, z_{1}\right)}=\iint F_{D\left(z_{1}, z_{0}\right)} P_{\left(x_{0}, y_{0}, z_{0}\right)} S_{\left(x_{0}, y_{0}, z_{0}\right)} d x d y \\
z_{2}: \quad B_{D\left(x_{2}, y_{2}, z_{2}\right)}=\iint F_{D\left(z_{2}, z_{1}\right)} B_{D\left(x_{1}, y_{1}, z_{1}\right)} S_{\left(x_{1}, y_{1}, z_{1}\right)} d x d y \\
\vdots \\
Z_{m}: B_{D i d\left(z_{m}\right)}=\iint F_{D\left(z_{m}, z_{m-1}\right)} B_{D\left(x_{m_{1}}, y_{m-1}, z_{m-1}\right)} S_{i\left(x_{m-1}, y_{m-2}, z_{m-1}\right)} d x d y
\end{gathered}
$$

where $S_{D\left(x_{0}, y_{0}, z_{0}\right)}$ is the receiver operator at the surface, and $S_{\left.\mathrm{x}_{1,1} \mathrm{y}_{1}, \mathrm{z}_{1}\right)} \cdots S_{\left(x_{m-1}, y_{m-1}, z_{m-1}\right)}$ are the grid operators at the subsurface interfaces. The value of the grid operators is 1 at the grid point and 0 at other positions. The size of the grid, $b$, should meet the spatial sampling condition,

$$
b \leqslant V_{i} / 2 f_{d}
$$

where $V_{i}$ represents the layer velocity, and $f_{\mathrm{d}}$ denotes the dominant frequency of the pressure wave.

Note that the area of integration in equations (16) and (17) defines the focusing aperture, or imaging aperture. Depending on the subsurface conditions, it can be a small area representing the target area, or the area within the whole boundary of the model. The bigger 
the area is, the more demanding the calculation will be.

The focal source beams $B_{S d i\left(z_{m}\right)}$ can be calculated following the same procedure. In this way, we determine both the detector beams $B_{\operatorname{Did}\left(z_{m}\right)}$ and the source beams $B_{\operatorname{Sdi}\left(z_{m}\right)}$. Hence, the resolution function can be constructed according to equation (9).

\section{Numerical tests on accuracy and validity}

In the previous section, we have presented a double-focusing numerical procedure to calculate the migration resolution function for layered media. We now perform some numerical tests to assess its accuracy and validity. For this, we designed a swath template for data acquisition (Table 1), and test the method using three models (Figure 4).

The parameters of the swath template are given in Table 1. It consists of one source line of 8 shots, and 8 receiver lines of 192 receivers, with the shot and receiver interval both set to $100 \mathrm{~m}$. The template rolls for 20 times in the in-line direction (x-direction or receiver line direction) with a roll interval of $200 \mathrm{~m}$, representing a shot line interval of $200 \mathrm{~m}$. The template rolls 5 times in the cross-line direction (y-direction or shot line direction) with a roll interval of $800 \mathrm{~m}$. This gives rise to a survey area of $3300 \mathrm{~m} \times 4600 \mathrm{~m}(x$ and $y)$. A wavelet with $30 \mathrm{~Hz}$ dominant frequency is used for the tests. The scale of the 3D models is $3300 \mathrm{~m} \times 4600 \mathrm{~m} \times$ $3000 \mathrm{~m}(x, y$ and $z)$, and the target point locates in the bottom-center of the model.

The first model is a homogenous medium with velocity 3000m/s (Model 1, Figure 4a). The resolution function for this model can be directly calculated using equations (10) to (13), which serves as the standard solution for comparison. The second model is a three-layer medium, but each layer has the same velocity of $3000 \mathrm{~m} / \mathrm{s}$ (Model 2, Figure 4b). The numerical procedure presented in the previous section [equations (16) and (17)] is applied to this model in order to test the accuracy of the numerical scheme, which should yield the same results as model 1 if our numerical method is valid. The third model consists of a single dipping layer with a dip angle of $20^{\circ}$, the average velocity across the interface is about $3000 \mathrm{~m} / \mathrm{s}$ (Model 3, Figure 4c): the velocity of the upper layer is $2700 \mathrm{~m} / \mathrm{s}$ whilst the velocity of the lower is $3333 \mathrm{~m} / \mathrm{s}$. This model is used to evaluate the effects of dip and layering on the resolution function.

Figure 5 shows the horizontal slices of the resolution functions calculated by the doublefocusing numerical procedure in the previous section for the three models in Figure 4. As 
expected, the results of Figure 5a and 5b are almost identical, confirming that the numerical procedure presented is very accurate and our recursive approach is valid. In contrast, the result for the dipping model (Figure 5c) is clearly different, as the main lobe is much wider in the $\mathrm{x}$-direction, turning from a circle into an ellipse. Furthermore, quantitative analysis of the cross sections along the $\mathrm{x}-\mathrm{z}$ (dip plane) and y-z (strike plane), as shown in Figure 6, reveals a $10 \mathrm{~m}$ difference in resolution (Table 2). Note that here the resolution is estimated as the half width of the main lobe in Figure 5. This indicates that wavefield extrapolation is necessary in layered media, and the use of homogenous solutions for layered media may introduce severe error. Our numerical extrapolation scheme provides an effective way to evaluate the effects of layering and dip, and can be used for optimizing spatial resolution and acquisition design.

\section{An example of application to acquisition design}

The above algorithm can be applied to evaluate the spatial resolution of different model geometries as well as different acquisition systems. During seismic experiments, the choice of model geometry is important, however the construction of a physical model is time-consuming (Wang et al., 2007). Our algorithm can help in optimizing model geometry before the model is constructed. Similarly, in 3D data acquisition, spatial resolution is a key parameter for optimizing the acquisition geometry. Here we use a 3D model to illustrate these applications.

As shown in Figure 7, the 3D model has a scale-up dimension of $4000 \mathrm{~m} \times 4000 \mathrm{~m} \times$ $3000 \mathrm{~m}(x, y$ and $z$ ), and it consists of three layers with velocities of $2900 \mathrm{~m} / \mathrm{s}, 2500 \mathrm{~m} / \mathrm{s}$ and $3200 \mathrm{~m} / \mathrm{s}$ respectively. The first two layers are dipping layers with the same strike direction but opposite dip direction; the dip angles are $10^{\circ}$ and $5^{\circ}$ respectively; and the depths of the middle point of the interfaces are at $800 \mathrm{~m}$ and $1500 \mathrm{~m}$, respectively. The same swath template as in Table 1 is used and is placed at the top horizontal surface. The template rolls 4 times in the cross-line direction at an interval of $800 \mathrm{~m}$ and 5 times in the in-line direction at an interval of $200 \mathrm{~m}$.

We place the diffractor at the bottom-center position and its $(x, y, z)$ coordinates are (2000m, 2000m, 3000m), respectively. To evaluate the resolution for different dip angles, we set the dip angles of the imaging plane as $0^{\circ}$ (horizontal), $10^{\circ}, 30^{\circ}$ and $60^{\circ}$, as shown in Figure 7, but keep the strike in the crossline direction (y-direction). A wavelet with a dominant frequency of $30 \mathrm{~Hz}$ is used.

Figure 8 shows the horizontal slices of the resolution functions corresponding to the 
imaging planes at the four dip angles. Comparison of Figure 8a with 8b reveals that there is little difference between the resolution functions corresponding to the imaging planes of $0^{\circ}$ and $10^{\circ}$ dip angles. However, for a dip angle of $30^{\circ}$, the main lobe of the resolution function becomes significantly wider in the inline $x$-direction (the dip direction, Figure 8c) whilst unchanged in $y$-direction (the strike direction), compared with Figures 8a and 8b. When the dip angle of the imaging plane reaches $60^{\circ}$, as shown in Figure 8d, the main lobe of the resolution function becomes even wider in the $x$-direction, but only slightly changed in the $y$-direction. Details of these changes in resolution can be obtained by analyzing the $\mathrm{x}-\mathrm{z}$ and $\mathrm{y}-\mathrm{z}$ cross sections, and the results are listed in Table 3. As the dip increases, the resolution of migration is significantly reduced in the dip direction, whilst the resolution along the strike direction is almost unchanged.

The above example shows that for a given acquisition geometry, the double focusing algorithm for layered media can be used to quantify the spatial resolution of the migrated image of subsurface structures, and hence to help acquisition design in 3D seismic surveys.

\section{Discussion and conclusions}

Currently there are no effective methods for calculating the resolution function for layered media. Instead, the algorithm for a homogeneous medium is often used for estimating spatial resolution in layered media. This can only serve as an approximation. In this paper, we have aimed to fill that gap.

Based on Berkhout's double focusing concept, we have developed a numerical procedure for extrapolating the wavefield and calculating the resolution function of a point diffractor on an imaging plane in layered media. The essential part of this procedure is to treat each individual layer in the layered media as a homogeneous medium, and the algorithm for the homogeneous medium can then be used recursively to extrapolate the wavefield, layer by layer, through the interfaces. The key step in this procedure is the calculation of the focal detector and source beams through forward and inverse extrapolation of the wavefield using Rayleigh or Kirchhoff integrations. The Rayleigh integration is used for planar interfaces, whilst the Kirchhoff integration is used for curved interfaces.

Theoretical speaking, any migration method can be used to make an image volume on synthetic dataset generated from a diffractor model for complex media, and this image volume may then be regarded as the digital resolution function of the corresponding complex media. The main advantage using Berkhout's approach with integrated solutions is that it not 
only gives rise to an analytical expression of the resolution function, as in equation (9), but also offers a straightforward and efficient solution to the problem.

We have calculated the resolution functions corresponding to different imaging planes with different dip angles. Through the calculation of spatial resolution, we are able to analyze the effect of subsurface geological structures on the potential resolving power of seismic migration under a given acquisition system. This is illustrated using a three-layer physical model with different model geometries. As the dip angle increases, the resolution decreases in the dip direction whilst is almost unchanged in the strike direction. We conclude from the test results that the double-focusing numerical procedure presented in this paper is sufficiently accurate for layered stratified media, and can be applied to quantify the spatial resolution of migrated image of the subsurface structures and in turn to optimize 3D acquisition geometries.

\section{Acknowledgements}

We thank CNPC Geophysical Key Laboratory for permission for publishing this work. We are also grateful to the support offered by BGP-CNPC offshore.

\section{Appendix. Wave field extrapolation for the dipole source}

As shown in Figure 2, we consider the wave field excited at point B propagating directly to plane $S_{1}$. The wave field on plane $S_{1}$ for $k r>>1$ can be written as:

$$
P_{S 1}=\frac{j k Z_{\mathrm{B}}}{2 \pi r^{2}} e^{-j k r_{B}}
$$

With Rayleigh integration II (Berkhout 1985), the wave field at point A extrapolated from plane $\mathrm{S}_{1}$ can be formulated as:

$$
P_{A 1}=-\frac{k^{2} Z_{A} Z_{B}}{4 \pi^{2}} \iint_{S 1} \frac{1}{r_{A}{ }^{2} r_{B}{ }^{2}} e^{-j k\left(r_{A}+r_{B}\right)} d x d y
$$

Using polar coordinates, where, $\rho^{2}=\mathrm{x}^{2}+\mathrm{y}^{2},{r_{A}}^{2}=\rho^{2}+\mathrm{z}_{\mathrm{A}}{ }^{2},{r_{B}}^{2}=\rho^{2}+\mathrm{z}_{B}{ }^{2}$, $d x d y=\rho d \rho d \alpha$, and considering $\oint d \alpha=2 \pi$, equation (A.2) can be written as: 


$$
P_{A 1}=\frac{k^{2} Z_{A} Z_{B}}{4 \pi} \int_{0}^{L} \frac{1}{r_{A}{ }^{2} r_{B}{ }^{2}} e^{-j k\left(r_{A}+r_{B}\right)} d \rho^{2},
$$

which can be further reformulated into:

$$
P_{A 1}=-\frac{j k Z_{A} Z_{B}}{2 \pi} \int_{0}^{L} \frac{1}{r_{A} r_{B}\left(r_{A}+r_{B}\right)} d e^{-j k\left(r_{A}+r_{B}\right)}
$$

Integrating equation (A.4) by parts gives:

$$
P_{A 1}=\frac{j k Z_{A} Z_{B}}{2 \pi}\left(G+\int_{0}^{L} H e^{-j k\left(r_{A}+r_{B}\right)} d \rho^{2}\right)
$$

where

$$
\begin{gathered}
G=\frac{e^{-j k\left(r_{A}+r_{B}\right)}}{r_{A} r_{B}\left(r_{A}+r_{B}\right)} ; \text { and } \\
H=\frac{1}{r_{A}{ }^{2} r_{B}{ }^{2}\left(r_{A}+r_{B}\right)}+\frac{1}{2 r_{A}^{3}\left(r_{A}+r_{B}\right)^{2}}+\frac{1}{2 r_{B}^{3}\left(r_{A}+r_{B}\right)^{2}}
\end{gathered}
$$

Considering $r \gg>1$, and comparing $H$ with the integrand, it follows that

$$
H<<\frac{1}{r_{A}^{2} r_{B}^{2}}
$$

which indicates that the second term in equation (A.5) is much smaller than the first term, and may be omitted. Therefore, equation (A.5) may be simplified as:

$$
\begin{aligned}
P_{A 1} & \approx-\frac{j k z_{A} z_{B}}{2 \pi} G=-\left.\frac{j k z_{A} z_{B}}{2 \pi r_{A} r_{B}\left(r_{A}+r_{B}\right)} e^{-j k\left(r_{A}+r_{B}\right)}\right|_{0} ^{L}= \\
& =\frac{j k}{2 \pi\left(z_{A}+z_{B}\right)}\left[e^{-j k\left(z_{A}+z_{B}\right)}-\frac{z_{A} z_{B}\left(z_{A}+z_{B}\right)}{r_{A m} r_{B m}\left(r_{A m}+r_{B m}\right)} e^{-j k\left(r_{A m}+r_{B m}\right)}\right],
\end{aligned}
$$

which is equation (15).

\section{References}

Berkhout A J 1985 Seismic Migration: Imaging of acoustic energy by wavefield extrapolation Third Edition: Elsevier Science Publication

Berkhout A J 1997 Pushing the limits of seismic imaging, part I: Prestack migration in terms 
of double dynamic focusing Geophysics 62 937-953

Berkhout A J, Ongkiehong O, Volker A W F and Blacquiere G 2001 Comprehensive assessment of seismic acquisition geometries by focal beams-Part I: Theoretical considerations Geophysics 66 911-917

Beylkin G, Oristaglio M and Miller D 1985 Spatial resolution of migration algorithms Proceeding of $14^{\text {th }}$ Internat. Symp. On Acoustical Imaging 155-167

Chen J and Schuster G T 1999 Resolution limits of migrated images Geophysics 64 1046-1053

Ma Z, Jin S, Chen J and Wang H 2002 Quantitative estimation of seismic imaging resolution 72nd Ann. Internat. Mtg: Soc. of Expl. Geophys. Expanded Abstracts pp 2281-2284.

Vermeer G J O 1999 Factors affecting spatial resolution Geophysics 64 942-953

Volker A W F, Blacquiere G, Berkhout A J and Ongkiehong O 2001 Comprehensive assessment of seismic acquisition geometries by focal beams -- Part II: Practical aspects and examples Geophysics 66 918-931

Von Seggern D 1994 Depth-imaging resolution of 3-D seismic recording patterns Geophysics 59 564-576.

Wang, S., Li, X.-Y., Zhong, P., Di, B. and Wei, J., 2007. Physical modeling studies of 3-D P-wave seismic for fracture detection. Geophysical Journal International, 168, 745-756.

Wapenaar C P A1997 3-D migration of cross-spread data: Resolution and amplitude aspects. Geophysics 62 1220-1225

\section{Table captions}

Table 1. Parameters of the swath template used for data acquisitions.

\begin{tabular}{|l|l|}
\hline Shot line interval: $200 \mathrm{~m}$ & Receiver line interval: $200 \mathrm{~m}$ \\
\hline Shot interval: $100 \mathrm{~m}$ & Receiver interval: $100 \mathrm{~m}$ \\
\hline Minimum offset: $200 \mathrm{~m}$ & Maximum offset: $2500 \mathrm{~m}$ \\
\hline Number of shot line: 1 & Number receiver lines: 8 \\
\hline Number of channels: 192 & Number shots: 8 \\
\hline Nominal fold: 12 & Nominal bin size: $50 \mathrm{mx} 50 \mathrm{~m}$ \\
\hline
\end{tabular}

Table 2. Quantitative analysis of the resolution for the three models in Figure 4. The resultion is measured as the half width of the main lobe in Figure 6. 


\begin{tabular}{|l|l|l|l|}
\hline Models & 1 & 2 & 3 \\
\hline x-direction(m) & 68 & 68 & 74 \\
\hline y-direction(m) & 62 & 62 & 64 \\
\hline
\end{tabular}

Table 3. Comparison of the resolution in the dip and strike directions for imaging planes with different dip angles.

\begin{tabular}{|l|l|l|l|l|}
\hline Dip angle $\left(^{\circ}\right)$ & 0 & 10 & 30 & 60 \\
\hline Resolution in the dip direction (m) & 71 & 72 & 82 & 142 \\
\hline Resolution in the strike direction (m) & 109 & 109 & 109 & 109 \\
\hline
\end{tabular}




\section{Figure captions}

Figure 1. A schematic diagram showing the calculation of focal detector and source beams for a point diffractor in a homogeneous medium. $\mathrm{d}\left(0,0, \mathrm{z}_{\mathrm{d}}\right)$ is the target diffractor point, whereas $i$ is the imaging point that varies around d. For calculating the focal detector beam, $r_{d}$ represents the distance between the diffractor and the detector $S_{D} ; \varphi_{d}$ is the emergent angle of the diffractor; $r$ is the distance between the imaging point and the detector $\mathrm{S}_{\mathrm{D}} ; \varphi$ is the emergent angle of the imaging point. Note that for calculating the focal source beam, $r_{d}$ represents the distance between the diffractor and the source $\mathrm{S}_{\mathrm{s}}$, and so on.

Figure 2. A schematic diagram illustrating the procedure of wavefield extrapolation with Rayleigh integration. The source is located at point B; A represents a point in the medium; $\mathrm{S}_{1}$ represents a reference plane for wave field extrapolation; and the extrapolation aperture is $2 \mathrm{~L}$.

Figure 3. A schematic diagram illustrating the procedure to calculate the focal detector beam in layered media. (a) Forward wavefield extrapolation recursively through the layer interfaces upward from the subsurface diffractor to the detectors on the surface; (b) inverse wavefield extrapolation from the surface detectors to the imaging points on the subsurface. The imaging point varies around the diffractor point on the imaging plane.

Figure 4. The three models used for evaluating the accuracy of the numerical algorithm. (a) a homogenous medium with velocity $3000 \mathrm{~m} / \mathrm{s}$; (b) a three-layer medium, where each layer has the same velocity of $3000 \mathrm{~m} / \mathrm{s}$; (3) a single dipping layer with a dip angle of $20^{\circ}$, the average velocity across the interface is about $3000 \mathrm{~m} / \mathrm{s}$.

Figure 5. Horizontal slices of the resolution functions for the three models in Figure 4.

Figure 6. (a) $x-z$ and (b) $y-z$ cross sections of the resolution function in Figure $5 c$ for the dipping layer model. 
Figure 7. A 3D model for illustrating the application of the algorithm. The 3D model has a dimension of $4000 \mathrm{~m} \times 4000 \mathrm{~m} \times 3000 \mathrm{~m}(\mathrm{x}, \mathrm{y}, \mathrm{z})$, and it consists of three layers with velocities of $2900 \mathrm{~m} / \mathrm{s}, 2500 \mathrm{~m} / \mathrm{s}$ and $3200 \mathrm{~m} / \mathrm{s}$ respectively. The first two layers are dipping layers with the same strike direction but opposite dip direction; the dip angles are $10^{\circ}$ and $5^{\circ}$ respectively. A diffractor is placed at the bottom-center position with $(\mathrm{x}, \mathrm{y}, \mathrm{z})$ coordinates of (2000m,2000m,3000m), respectively. For imaging planes with dip angles of $0^{\circ}$ (horizontal), $10^{\circ}, 30^{\circ}$ and $60^{\circ}$ are selected to evaluate the resolution of double focus migration.

Figure 8. The horizontal slices of the resolution functions for the four imaging planes with dip angles of: (a) $0^{\circ}$ (horizontal), (b) $10^{\circ}$, (c) $30^{\circ}$ and (d) $60^{\circ}$. 


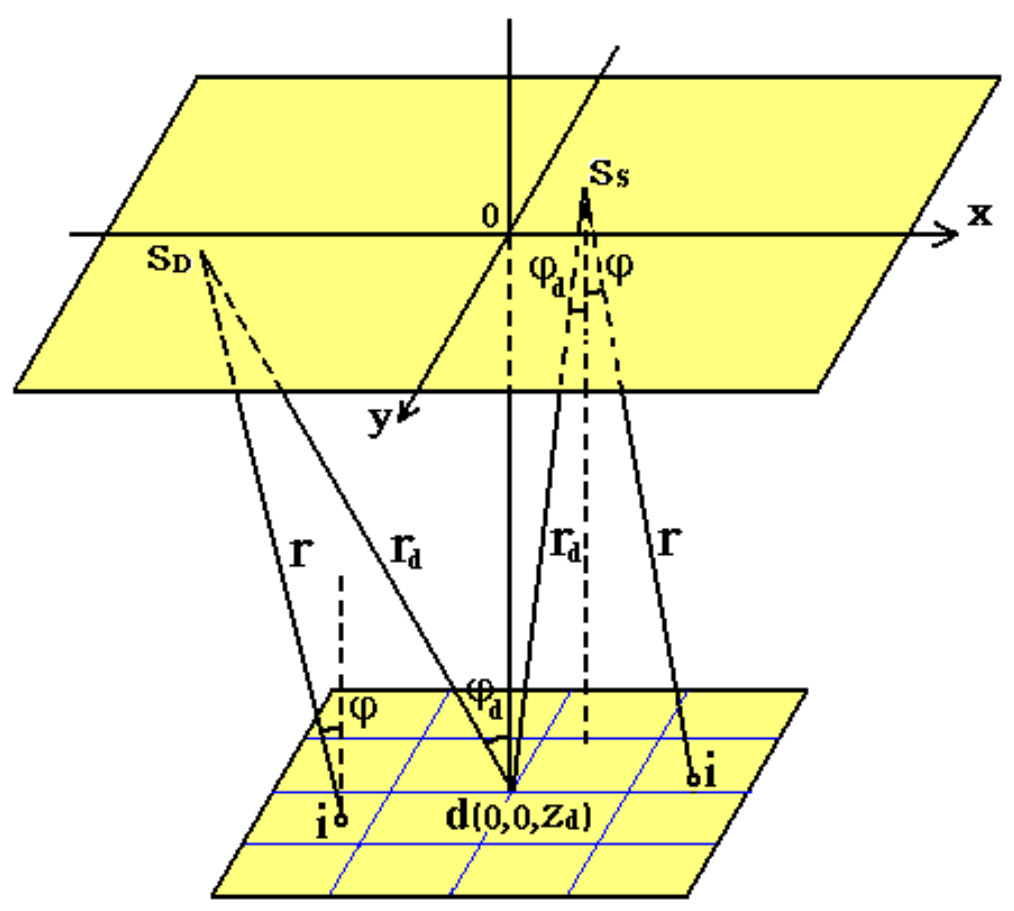

Figure 1. A schematic diagram showing the calculation of focal detector and source beams for a point diffractor in a homogeneous medium. $d\left(0,0, \mathrm{z}_{\mathrm{d}}\right)$ is the target diffractor point, whereas $i$ is the imaging point that varies around d. For calculating the focal detector beam, $r_{d}$ represents the distance between the diffractor and the detector $\mathrm{S}_{\mathrm{D}} ; \varphi_{\mathrm{d}}$ is the emergent angle of the diffractor; $r$ is the distance between the imaging point and the detector $\mathrm{S}_{\mathrm{D}} ; \varphi$ is the emergent angle of the imaging point. Note that for calculating the focal source beam, $r_{d}$ represents the distance between the diffractor and the source $\mathrm{S}_{\mathrm{S}}$, and so on. 


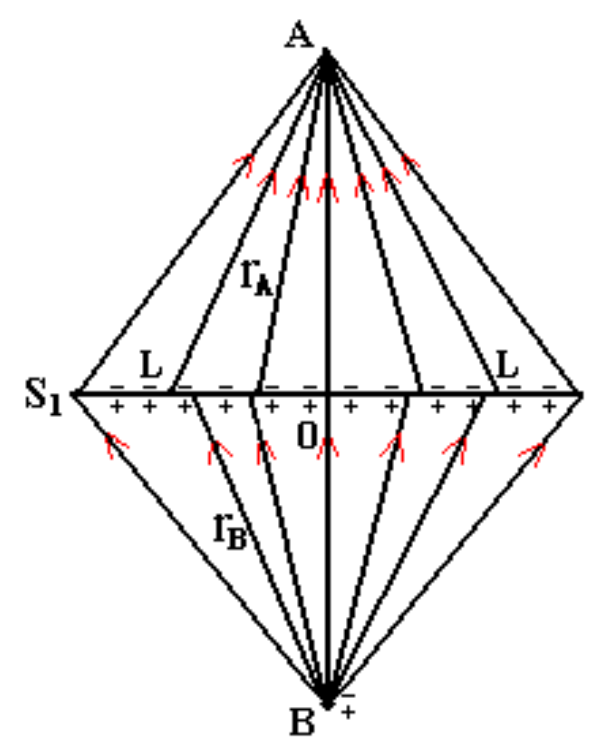

Figure 2. A schematic diagram illustrating the procedure of wavefield extrapolation with Rayleigh integration. The source is located at point B; Position A represents a point in the medium; $S_{1}$ represents a reference plane for wave field extrapolation; and the extrapolation aperture is $2 \mathrm{~L}$.

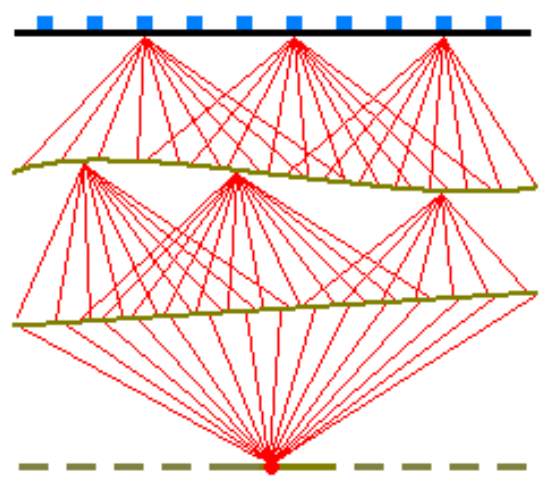

(a) Forward extrapolation

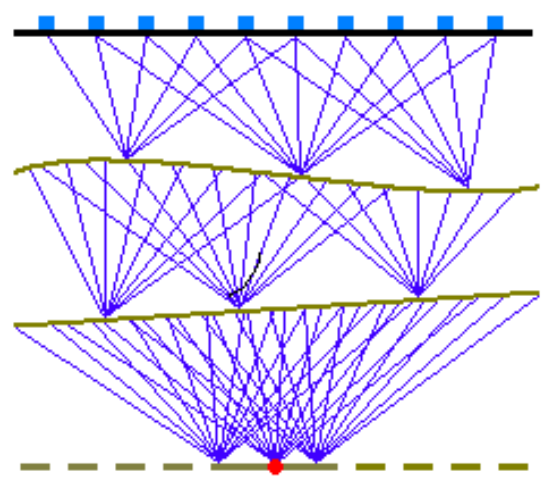

(b) Inverse extrapolation

Figure 3. A schematic diagram illustrating the procedure to calculate the focal detector beam in layered media. (a) Forward wavefield extrapolation recursively through the layer interfaces upward from the subsurface diffractor to the detectors on the surface; (b) inverse wavefield extrapolation from the surface detectors to the imaging points on the subsurface. The imaging point varies around the diffractor point on the imaging plane. 


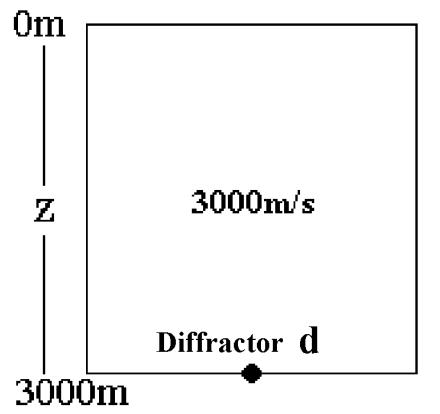

(a) Model 1

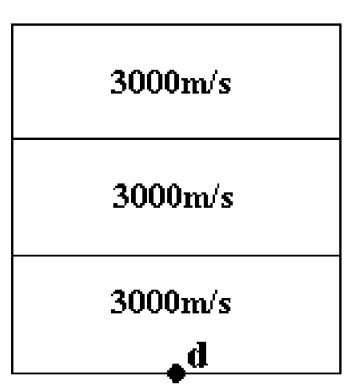

(b) Model 2

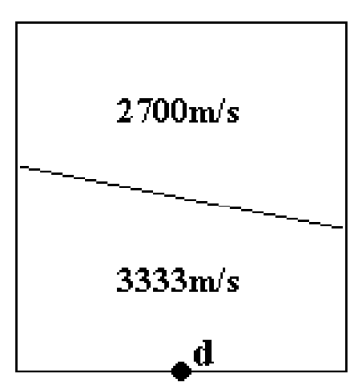

(c) Model 3

Figure 4. The three models used for evaluating the accuracy of the numerical algorithm. (a) a homogenous medium with velocity $3000 \mathrm{~m} / \mathrm{s}$; (b) a three-layer medium, where each layer has the same velocity of $3000 \mathrm{~m} / \mathrm{s}$; (3) a single dipping layer with a dip angle of $20^{\circ}$, the average velocity across the interface is about $3000 \mathrm{~m} / \mathrm{s}$.

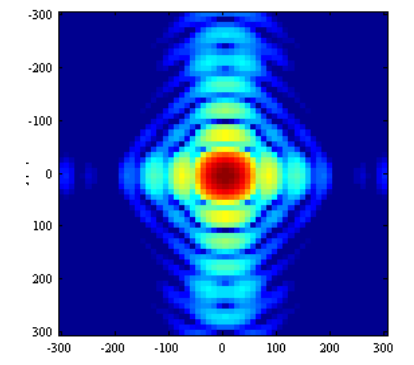

(a) Model 1

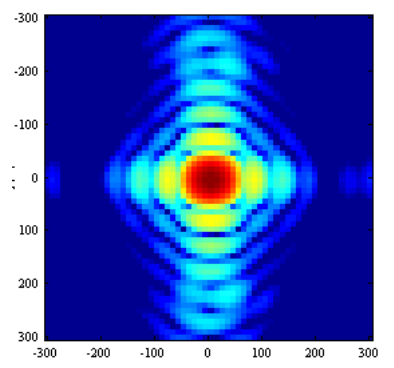

(b) Model 2

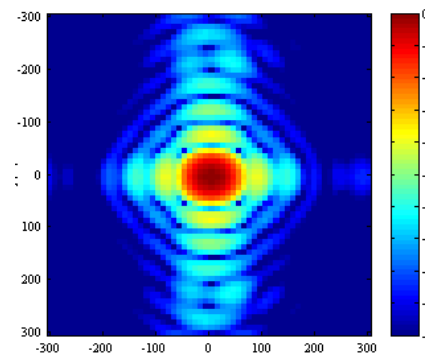

(c) Model 3

Figure 5. Horizontal slices of the resolution functions for the three models in Figure 4. 


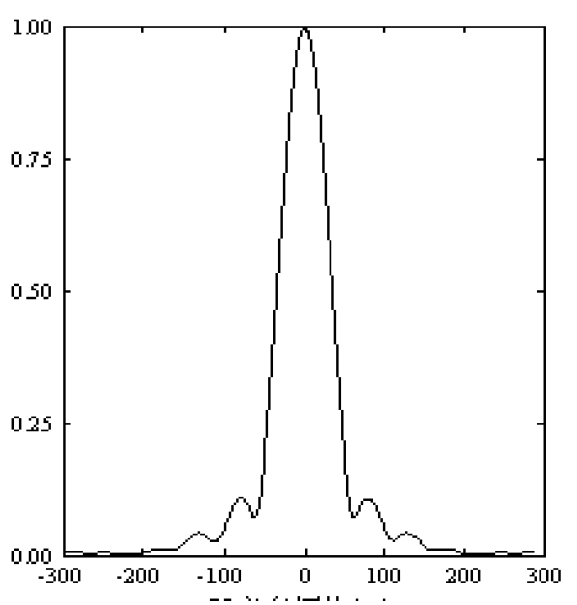

(a) $\mathrm{x}-\mathrm{z}$ section

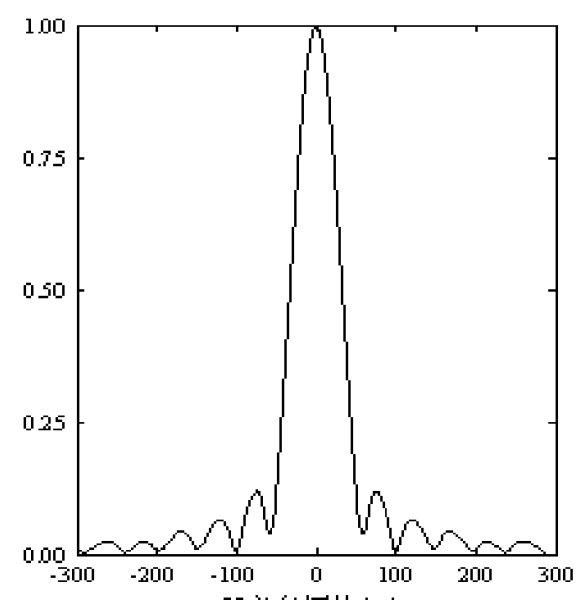

(b) y-z section

Figure 6. (a) $x-z$ and (b) $y-z$ cross sections of the resolution function in Figure $5 c$ for the dipping layer model.

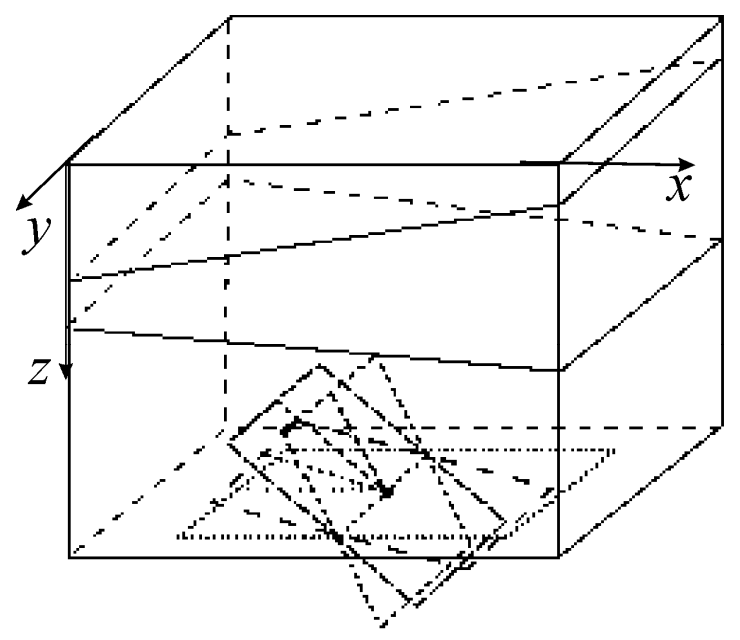

Figure 7. A 3D model for illustrating the application of the algorithm. The 3D model has a dimension of $4000 \mathrm{~m} \times 4000 \mathrm{~m} \times 3000 \mathrm{~m}(\mathrm{x}, \mathrm{y}, \mathrm{z})$, and it consists of three layers with velocities of $2900 \mathrm{~m} / \mathrm{s}, 2500 \mathrm{~m} / \mathrm{s}$ and $3200 \mathrm{~m} / \mathrm{s}$ respectively. The first two layers are dipping layers with the same strike direction but opposite dip direction; the dip angles are $10^{\circ}$ and $5^{\circ}$ respectively. A diffractor is placed at the bottom-center position with $(\mathrm{x}, \mathrm{y}, \mathrm{z})$ coordinates of $(2000 \mathrm{~m}, 2000 \mathrm{~m}, 3000 \mathrm{~m})$, respectively. For imaging planes with dip angles of $0^{\circ}$ (horizontal), $10^{\circ}, 30^{\circ}$ and $60^{\circ}$ are selected to evaluate the resolution of double focus migration. 


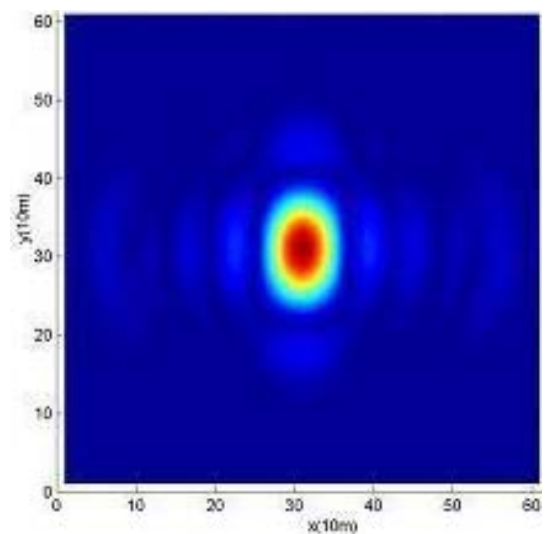

(a) $0^{0}$ (horizontal)

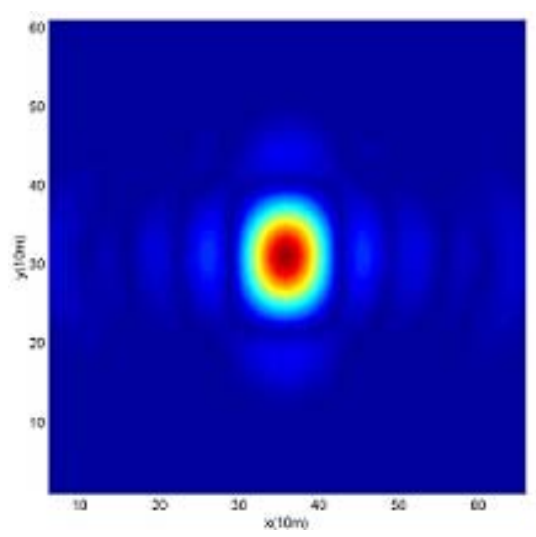

(c) $30^{0}$

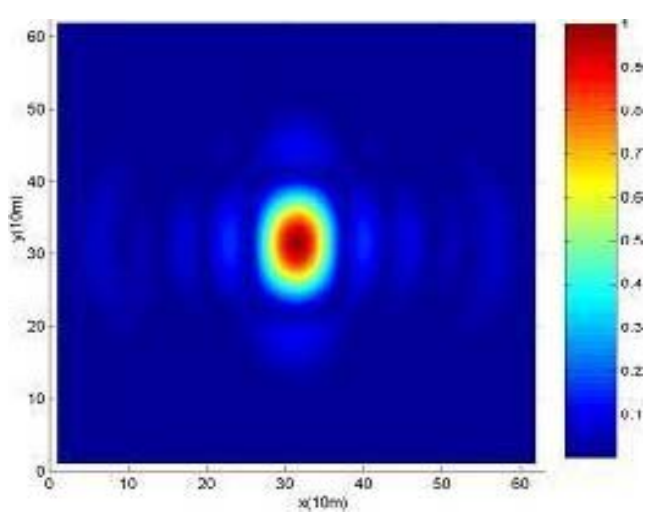

(b) $10^{0}$

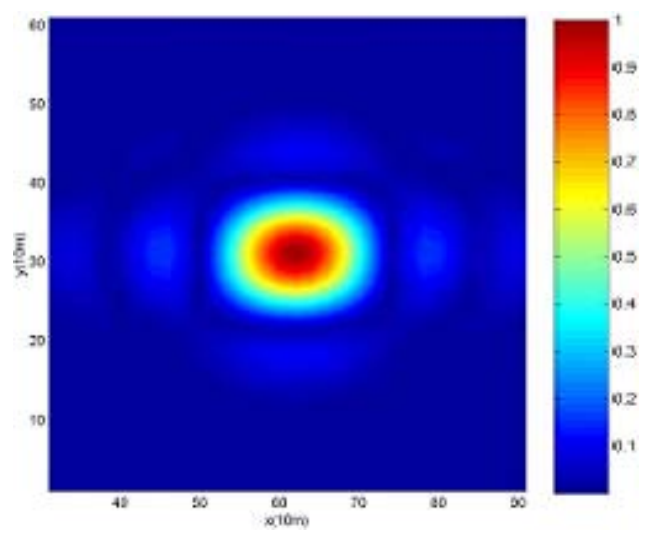

(d) $60^{\circ}$

Figure 8. The horizontal slices of the resolution functions for the four imaging planes with dip angles of: (a) $0^{\circ}$ (horizontal), (b) $10^{\circ}$, (c) $30^{\circ}$ and $\quad$ (d) $60^{\circ}$. 